\title{
Selenomonas noxia
}

National Cancer Institute

\section{Source}

National Cancer Institute. Selenomonas noxia. NCI Thesaurus. Code C86733.

A species of strictly anaerobic, Gram negative, crescent shaped bacteria assigned to the phylum Firmicutes. This species is esterase positive and cannot produce acid from sugars. S. noxia is found in human gingival spaces and is a cause of gingivitis and periodontal disease. 